\title{
Re-sequencing and optical mapping reveals misassemblies and real inversions on Corynebacterium pseudotuberculosis genomes
}

\author{
Thiago de Jesus Sousa ${ }^{1}{ }^{1}$, Doglas Parise ${ }^{1}$, Rodrigo Profeta ${ }^{1}$, \\ Mariana Teixeira Dornelles Parise ${ }^{1}$, Anne Cybelle Pinto Gomide ${ }^{1}$, Rodrigo Bentos Kato ${ }^{1}$, \\ Felipe Luiz Pereira ${ }^{2}$, Henrique Cesar Pereira Figueiredo $\mathbb{D}^{2}$, Rommel Ramos $\mathbb{D}^{3}$, \\ Bertram Brenig ${ }^{4}$, Artur Luiz da Costa da Silva $\mathbb{1}^{3}$, Preetam Ghosh ${ }^{5}$, Debmalya Barh 6 , \\ Aristóteles Góes-Neto ${ }^{1}$ \& Vasco Azevedo ${ }^{1 *}$
}

The number of draft genomes deposited in Genbank from the National Center for Biotechnology Information (NCBI) is higher than the complete ones. Draft genomes are assemblies that contain fragments of misassembled regions (gaps). Such draft genomes present a hindrance to the complete understanding of the biology and evolution of the organism since they lack genomic information. To overcome this problem, strategies to improve the assembly process are developed continuously. Also, the greatest challenge to the assembly progress is the presence of repetitive DNA regions. This article highlights the use of optical mapping, to detect and correct assembly errors in Corynebacterium pseudotuberculosis. We also demonstrate that choosing a reference genome should be done with caution to avoid assembly errors and loss of genetic information.

Next Generation Sequencing (NGS) platforms provide an exponential increase in the amount of data produced in a single assay (high-throughput data). This approach provided the scientific community with the ability to sequence more genomes at reduced costs. The NGS platforms perform the sequencing through different technologies, which were developed by different companies, such as 454 GS FLX system (Roche) ${ }^{1}$; Hiseq paired-end (Illumina) ${ }^{2}$; Ion Torrent PGM (Life Technologies) ${ }^{3}$; PacBio sequel system(Pacific Biosciences); and MinION (Oxford Nanopore) $)^{4}$. From these, thousands of genomic projects were created to sequence Bacteria, Archaea, and Eukarya species, viruses, and metagenomes ${ }^{5}$.

The main database of these sequences is GenBank maintained by the National Center for Biotechnology Information (NCBI), which in September 2018, contained 153,992 bacterial genomes, most of these being drafts, and only 11,103 sequences (7\%) were complete genome sequences. Furthermore, the complete sequences still might have misassemblies due to the presence of repetitive regions, such as ribosomal RNA (rRNA), transposases, phage regions, and plasmids ${ }^{6}$. These errors bias future studies and inferences, such as in comparative genomic or structural genomic analyses, and even ordering of phylogenetically related genomes ${ }^{7}$. Thus, obtaining a more precise and accurate complete genome sequence of an organism is fundamental to understanding its biological and evolutionary characteristics ${ }^{7}$.

The assembly problem persists even with the increase in the reads size, sequencing quality, and updates of $d e$ novo assembly algorithms. Another limiting factor to the increase of complete sequences is the lack of trained professionals. However, approaches to support this process have been gaining prominence ${ }^{8}$. For example, the use of $\mathrm{SSPACE}^{9}$ software to use paired-end reads to create a consensus sequence and perform scaffolding of contigs. Similarly, MapRepeat ${ }^{10}$ and riboSeed ${ }^{11}$ try to solve the repetitive region's problem.

${ }^{1}$ Institute of Biological Sciences, Federal University of Minas Gerais, Belo Horizonte, Minas Gerais, Brazil. ${ }^{2}$ National Reference Laboratory for Aquatic Animal Diseases (AOUACEN) of Ministry of Agriculture, Livestock and Food Supply, Federal University of Minas Gerais, Belo Horizonte, Minas Gerais, Brazil. 3Institute of Biological Sciences, Federal University of Pará, Belém, Pará, Brazil. ${ }^{4}$ Institute of Veterinary Medicine, University Göttingen, Göttingen, Germany. ${ }^{5}$ Department of Computer Science, Virginia Commonwealth University, Richmond, United States. ${ }^{6}$ Institute of Integrative Omics and Applied Biotechnology, Nonakuri West Bengal, India. *email: vasco@icb.ufmg.br 


\begin{tabular}{|c|c|c|c|c|c|c|c|}
\hline Strains & Sequencing & Reads & $\begin{array}{l}\text { Assembly } \\
\text { Software }\end{array}$ & $\begin{array}{l}\text { Length } \\
\text { (Mb) }\end{array}$ & $\begin{array}{l}\text { Mapped } \\
\text { reads (\%) }\end{array}$ & $\begin{array}{l}\text { Accession } \\
\text { number }\end{array}$ & Reference \\
\hline $1002 \mathrm{~B}$ & Ion PGM 200 bp & 739,755 & Mira v. 3.9.18 & 2.33511 & 99.70 & СР012837.1 & Mariano et al..$^{54}$ \\
\hline 29156 & Ion PGM $200 \mathrm{bp}$ & $1,400,026$ & Newbler v. 2.9 & 2.33865 & 98.02 & СР010795.1 & On this work \\
\hline I19 & Ion PGM $400 \mathrm{bp}$ & $1,255,111$ & Spades v. 3.6.0 & 2.33759 & 99.64 & CP002251.2 & On this work \\
\hline 31 & Ion PGM 400 bp & $1,394,211$ & SPAdes 3.6.0 & 2.40296 & 99.57 & СР003421.3 & Viana et al. ${ }^{55}$ \\
\hline 162 & Ion PGM 200 bp & $2,050,404$ & Newbler v. 2.9 . & 2.36587 & 98.00 & СР003652.2 & On this work \\
\hline 258 & Ion PGM 200 bp & 260,169 & Spades v. 3.6.0 & 2.36982 & 99.41 & СР003540.2 & Mariano et al..$^{56}$ \\
\hline CIP52.97 & Ion PGM $400 \mathrm{bp}$ & $1,427,084$ & Mira v. 3.9.18 & 2.36939 & 99.68 & CP003061.2 & On this work \\
\hline MB302 & Ion PGM 400 bp & $1,832,580$ & Newbler v. 2.9 & 2.36881 & 99.59 & CP021982.1 & Baraúna et al. ${ }^{57}$ \\
\hline $\mathrm{T} 1$ & Ion PGM 200 bp & $1,118,022$ & Newbler v. 2.9 & 2.3372 & 95.93 & СР015100.1 & Almeida et al..$^{58}$ \\
\hline MB11 & Ion PGM 200 bp & $6,753,458$ & Mira 4.0.2 & 2.36342 & 99.24 & СР013260.1 & Baraúna et al. ${ }^{59}$ \\
\hline
\end{tabular}

Table 1. Information on sequencing and assembling of strains.

In order to solve this assembly problem and to improve the generated data, we have the strategy of optical mapping, or Whole Genome Mapping (WGM), which is an approach that uses high-resolution restriction maps to generate the actual orientation of the organism's genome. It is the main method of large-scale genome analysis that provides complete visualization of the structural genome through a single image ${ }^{12}$. Optical mapping is based on the distance of the restriction sites for high precision map construction. This is a strategy in which data are obtained with greater precision since it is a physical result of the genome evaluation. This method, combined with the application of de novo assembly methodology, assists in the orientation of contigs ${ }^{13}$.

The technique of optical mapping was first developed by Schwartz and collaborators in 1993, with the purpose of studying the chromosomal gene ordering of Saccharomyces cerevisiae ${ }^{13}$. Samad et al., 1995, describe optical mapping as the novel approach for single-molecule DNA analysis using flowering microscopy to identify and estimate its size by the generated images ${ }^{14}$. Since then, several improvements have been added to the technique, especially in the images and algorithms for fragment size estimation ${ }^{15}$. Hence, WGM gained notoriety in several applications, such as in lineage typing in epidemic cases for clinical microbiology ${ }^{16,17}$; ordering of contigs generated by de novo assembly ${ }^{7}$; and in the study of inversions, insertions, deletions, duplications, and instability of bacterial genomes ${ }^{18,19}$. WGM has been successfully performed on very different types of organisms such as bacteria $^{20-22}$, fungi $i^{23}$, plants ${ }^{24-26}$, and mammals ${ }^{27}$.

Regarding the genomic assembly strategy, WGM is an additional method that allows the ordering of the contigs and thus provides a size estimation of the gaps and their positions. This combination of methods is called a hybrid approach to scaffolding assembly, and it is feasible to acquire a complete genome of high quality and accuracy.

The work carried out by Mariano and collaborators in 2016, updated the genome of Corynebacterium pseudotuberculosis $1002^{28}$ (later deposited as Cp1002B) using the optical mapping technique. C. pseudotuberculosis 1002B was the first organism of the Corynebacterium genus that had optical mapping applied in the detection and correction of assembly errors. From the results obtained by Mariano and collaborators in 2016, we decided to investigate another 10 genomes with this strategy, namely: C. pseudotuberculosis 29156 (Cp29156), C. pseudotuberculosis I19 (CpI19), C. pseudotuberculosis FRC41 (CpFRC41), C. pseudotuberculosis T1 (CpT1), C. pseudotuberculosis 31 (Cp31), C. pseudotuberculosis Cp162 (Cp162), C. pseudotuberculosis MB302 (CpMB302), C. pseudotuberculosis CIP52.97 (CpCIP52.97), C. pseudotuberculosis MB1 (CpMB11) and C. pseudotuberculosis 258 (Cp258) (Table 1). Among these genomes, 4 are strains from the biovar ovis and six from the biovar equi ${ }^{29}$.

The strategy is to make C. pseudotuberculosis the most used organism in genomic studies involving the Corynebacterium genus. Therefore, a total of 11 strains were selected from different hosts, isolation sites and distributed between ovis and equi biovars, so that complete genomes can be made available, well assembled, and updated by new sequencing. In this manner, this data can be explored with greater reliability by future comparative studies, intraspecific evolutionary relationship analyses.

\section{Results}

Sequencing and assembly. The strains deposited by our research group were either re-sequenced (i.e., Cp1002 (1002B), CpI19, Cp31, Cp162, Cp258, CpCIP52.97), or were first sequenced using the Ion Torrent PGM $^{\mathrm{TM}}$ platform (i.e., Cp29156, CpMB302, CpMB11, CpT1) (Table 1). Different software packages were used for de novo assembly (Table 1$)$.

Optical mapping analysis: biovar ovis strains. The strains Cp1002 (CP001809.2) and CpI19 (CP002251.1) (Fig. 1A,B) showed an inversion of approximately $1.6 \mathrm{Mb}$ and $1.22 \mathrm{Mb}$, respectively. It is observed in the regions flanking the first and third clusters of Ribosomal RNA in the CpI19 strain; while in Cp1002, it occurs between the first and fourth clusters. Figure 1A,B show the starting and ending points of the inversion, labeled as R1 and R2, respectively. The central block in Fig. 1 corresponds to the physical restriction map, while the upper and lower blocks represent the in silico restriction map generated by MapSolver ${ }^{\mathrm{TM}}$ software. The red regions of the central block (Fig. 1A,B) indicate that the same region exists in both the first version (Upper Block) and the updated version (Lower Block). Thus, they show that there was no significant loss between the compared versions in that region. 

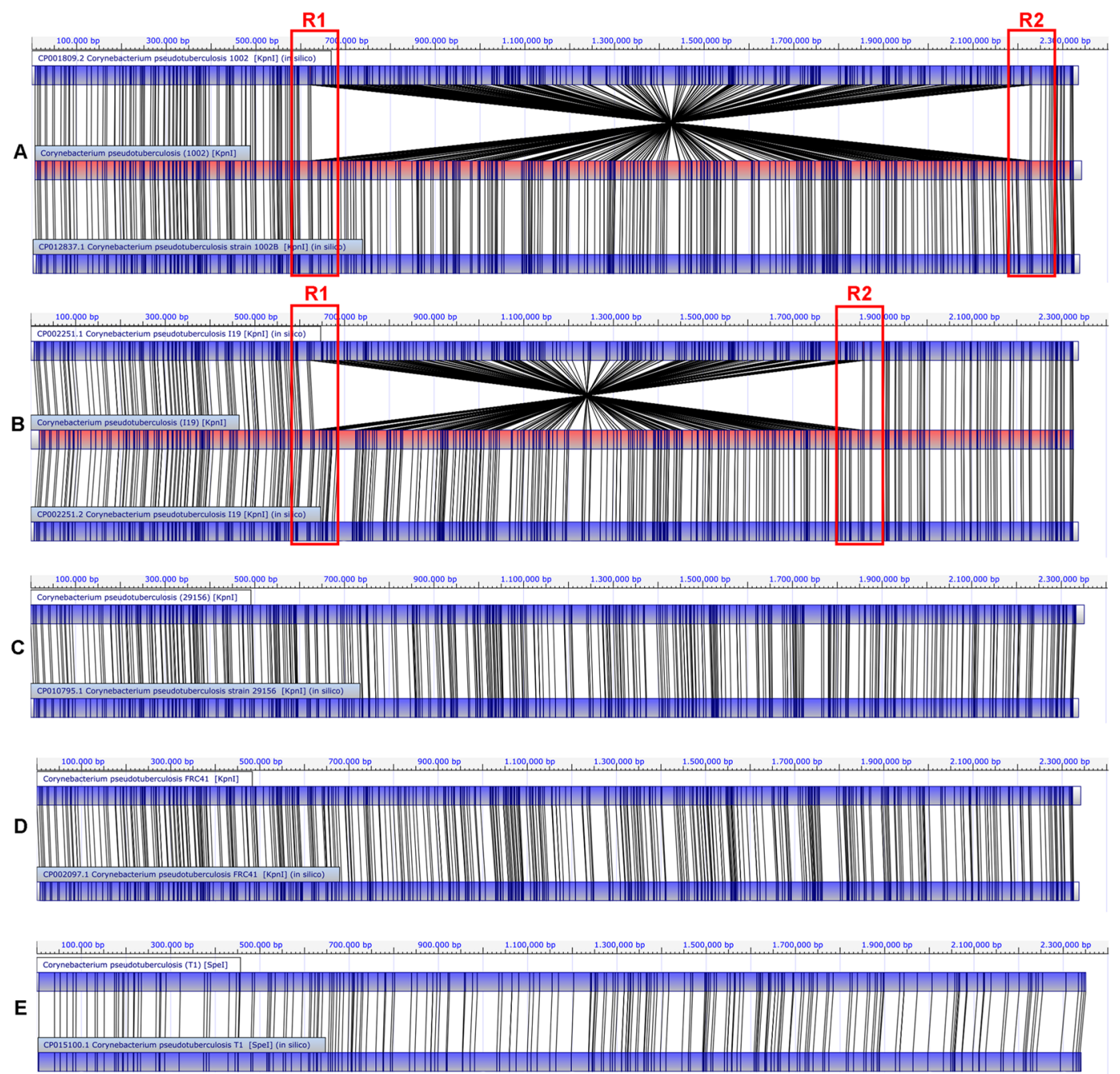

Figure 1. Optical map alignment of the selected ovis biovar strains. Comparisons between the first and the new version (when available), with C. pseudotuberculosis 1002 and 1002B (A); C. pseudotuberculosis I19 (B); C. pseudotuberculosis 29156 (C); C. pseudotuberculosis FRC41 (D); C. pseudotuberculosis T1 (E) are shown. R1 and R2 highlighted regions are events of inversion errors.

Strain CpFRC41 (CP002097.1) (Fig. 1D) showed a correct alignment of the restriction sites in the whole genome and thus, with no probable errors of assembly and ordering. Strains Cp29156 and CpT1 (Fig. 1C,E) were first sequenced in this work.

Optical mapping analysis: biovar equi strains. Figure 2A shows the alignment between the first (upper map) and the last (lower map) versions of the $\mathrm{CpCp} 31$ strain. The R1 region highlights the absence of corresponding restriction sites at the beginning of the genome. R2 region, in its turn, shows the absence of a chromosome region. This difference probably occurred due to errors in the assembly and gap closure process.

Worse problems were found in the previous version of Cp162 (Fig. 2B). The R1-labeled region, starting in $5^{\prime}$ end of the $d n a A$ gene, shows no similarity between the restriction site patterns. In the R2 region, there is no linear alignment between the sites, probably due to the absence of genes. According to the optical map, an error may have occurred in the ordering of contigs in the $\mathrm{R} 3$ region, where the segment should be in another region of the genome. R4 region shows a $\sim 0.85 \mathrm{Mb}$ inversion in the middle of the genome, located explicitly between two clusters of ribosomal RNA.

The CpMB11 strain presented an error of choice of chromosome initiation site, in which a segment close to $5^{\prime}$ end of the dnaA gene should be situated at the end (Fig. 2C, regions R1 and R2). The Cp258 strain did not present chromosomal inversions in the deposited genome. However, a misalignment of the restriction sites (Fig. 2D, region $\mathrm{R} 1$ ) is shown next to the origin of replication at the $5^{\prime}$ end of the genome. Also, a region containing the genes moaE, molB, molA, narI, narJ, narH, narG, narK, narT, moeY, moaC is absent in the deposited chromosome (Fig. 2D, R2). The same situation occurred with the strain CpCIP5297 (Fig. 2E, regions R1, and R2). Finally, as for the Cp29156 and CpT1 strains, it is the first sequencing of the CpMB302 strain (Fig. 2F). 

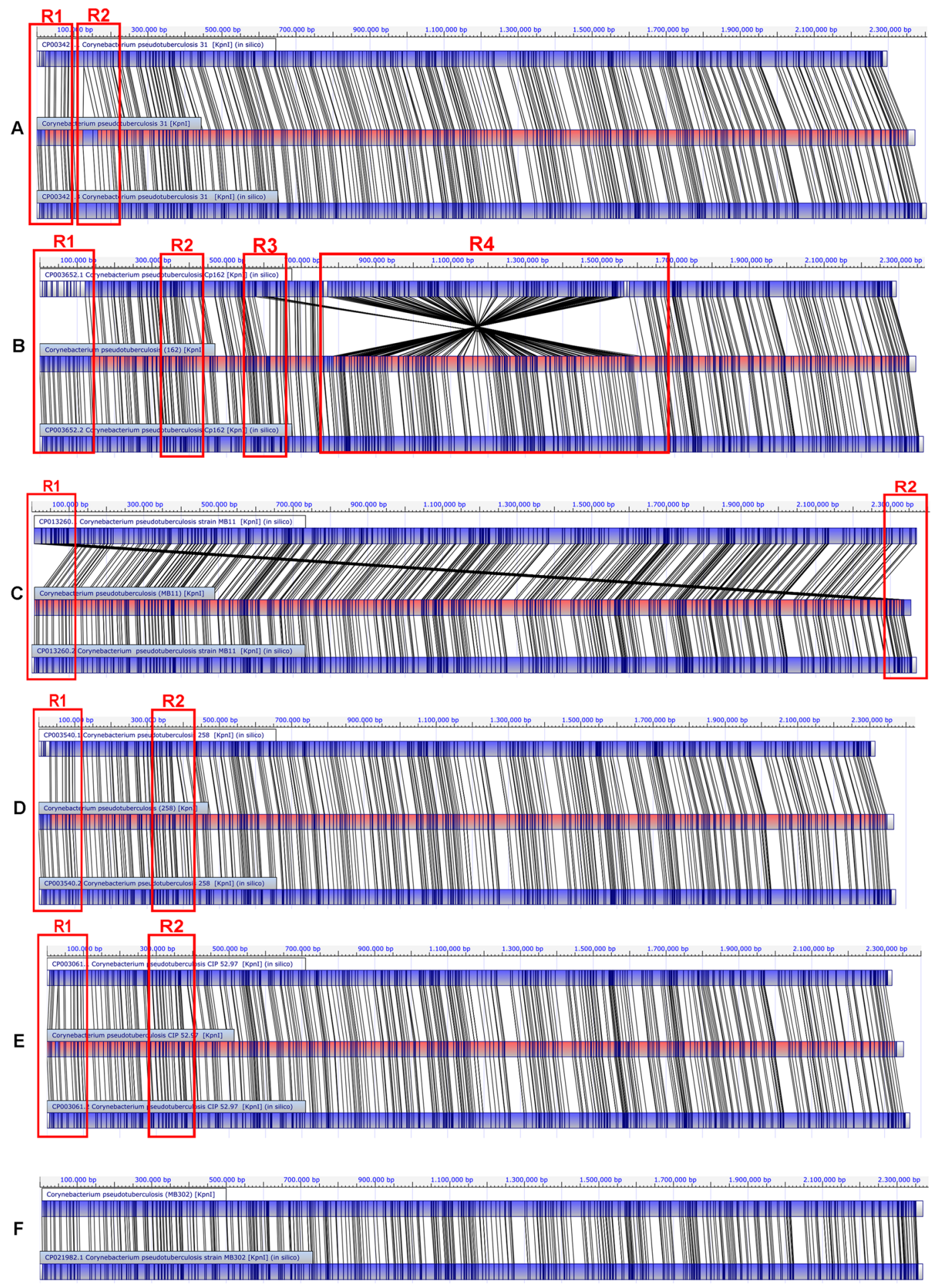

Figure 2. Optical map alignment of the selected equi biovar strains. Comparisons between the first and the new version (when available), with C. pseudotuberculosis 31 (A); C. pseudotuberculosis Cp162 (B); C. pseudotuberculosis MB11 (C); C. pseudotuberculosis 258 (D); C. pseudotuberculosis CIP52.97 (E); C. pseudotuberculosis $302(\mathbf{F})$ are shown.

Content and genomes plasticity. This analysis showed a reduction of $136 \mathrm{bp}$ in the total genome of the CpI19 strain, in addition to an increase of 34 CDSs and a reduction of 12 pseudogenes (Table 2). The updated version of strain $\mathrm{Cp} 1002^{28}$ showed a reduction of 6 bases in the total genome and a reduction in the number of annotated CDSs $(n=24)$ when compared with the older one. In this case, updating the annotation of genes identified as hypothetical protein might be the explanation. This comparison can be visualized in the map generated by BRIG, in which the last version is compared with the most updated one before the optical mapping. Strains 


\begin{tabular}{|c|c|c|c|}
\hline Isolates & Bases (bp) & CDS & Pseudogenes \\
\hline I1 $9^{1 \text { st }}$ & $2,337,730$ & 2,095 & 57 \\
\hline I1 $9^{\text {2nd }}$ & $2,337,594$ & 2,129 & 45 \\
\hline $1002^{2 \mathrm{nd}}$ & $2,335,113$ & 2,095 & 47 \\
\hline $1002 \mathrm{~B}^{1 \mathrm{st}}$ & $2,335,107$ & 2,071 & 43 \\
\hline $258^{\text {lst }}$ & $2,314,404$ & 2,088 & 46 \\
\hline $258^{\text {2nd }}$ & $2,369,817$ & 2,129 & 34 \\
\hline $162^{1 \mathrm{st}}$ & $2,293,464$ & 2,002 & 87 \\
\hline $162^{2 \mathrm{nd}}$ & $2,365,874$ & 2,099 & 43 \\
\hline $31^{1 \mathrm{st}}$ & $2,297,010$ & 2,063 & 46 \\
\hline $31^{3 \mathrm{rd}}$ & $2,402,956$ & 2,173 & 4 \\
\hline CIP52.971st & $2,320,595$ & 2,060 & 75 \\
\hline CIP52.972nd & $2,369,387$ & 2,187 & 62 \\
\hline
\end{tabular}

Table 2. Comparison between deposited and new version assembly of CpI19, Cp1002 (Cp1002B), Cp258, Cp162, Cp31, and CpCIP52.97 strains.
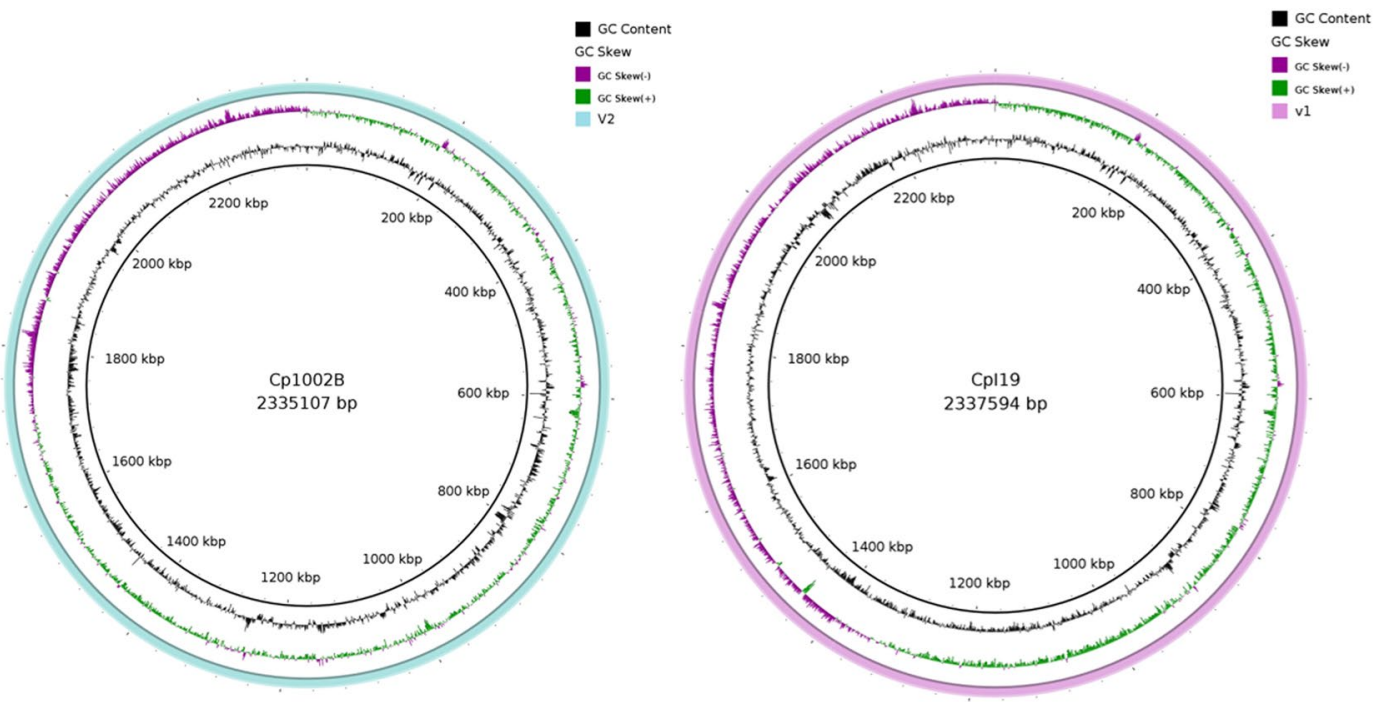

Figure 3. Comparative BRIG analysis of ovis biovar strains. Comparative genomic maps of the older versions (outermost circles) and their respective versions with optical map (inner black circles). (A) C. pseudotuberculosis 1002B. (B) C. pseudotuberculosis I19.

Cp1002B (Fig. 3A) and CpI19 (Fig. 3B) showed no gaps in the comparison, meaning that there were no relevant losses or gains among the versions.

The equi strains showed more changes among the genomes. In the Cp258 genome, obtained from the Ion Torrent PGM sequencing, an insert of $\sim 55 \mathrm{~kb}$ was added. A total of 41 new CDSs and a reduction of 12 pseudogenes were included (Table 2). This had not been represented within the first genome, which was obtained by SOLiD v3 sequencing. Differences can also be visualized on the genomic map (Fig. 4A), where the highlighted red genes are important and essential genes for the strain classification e.g., the operon nar, with the moaE, moaD, molB, molA, narI, narL, narG, narK, narT, moEY, mobA, moAc, moeA1 genes. Presence of genes coding for proteins such as Beta-lactamase, Vitamin K-dependent gamma-carboxylase, Heavy-metal-binding protein, Transposases, Type I restriction-modification system, and N-6 DNA Methylase is also important. Moreover, errors related to positioning and presence of CRISPR associated proteins were found among the assemblies.

The strain Cp162 also presented an increase of genomic content $(\sim 72 \mathrm{~kb})$. Ninety-seven CDS and a reduction of 44 pseudogenes were found (Table 2). Figure $4 \mathrm{~B}$ shows the absent regions of genes such as the complete cluster of the operon nar. Genes coding for $\mathrm{Fe}^{3+}$ dicitrate transport, ATP-binding protein FecE, Beta-lactamase, Vitamin K-dependent, gamma-carboxylase, Heavy-metal-binding protein, Phytoene dehydrogenase, CAAX protease self-immunity, Restriction endonuclease or methylase, Collagen-binding surface protein Cna-like, B-type domain protein, Membrane protein, ATP-dependent exonuclease, and several hypothetical proteins were also absent. A possible assembly error in the cluster of genes coding for CRISPR-associated proteins was found.

The new sequencing by Ion Torrent of the strain Cp31 resulted in the most significant increase in the gene content $(\sim 106 \mathrm{~kb})$ among the selected strains. An increase of $110 \mathrm{CDSs}$ and a reduction of 42 pseudogenes (Table 2) were detected. In Fig. 4C, we can highlight the inclusion of the corynephage with the tox gene of diphtheria toxin. 


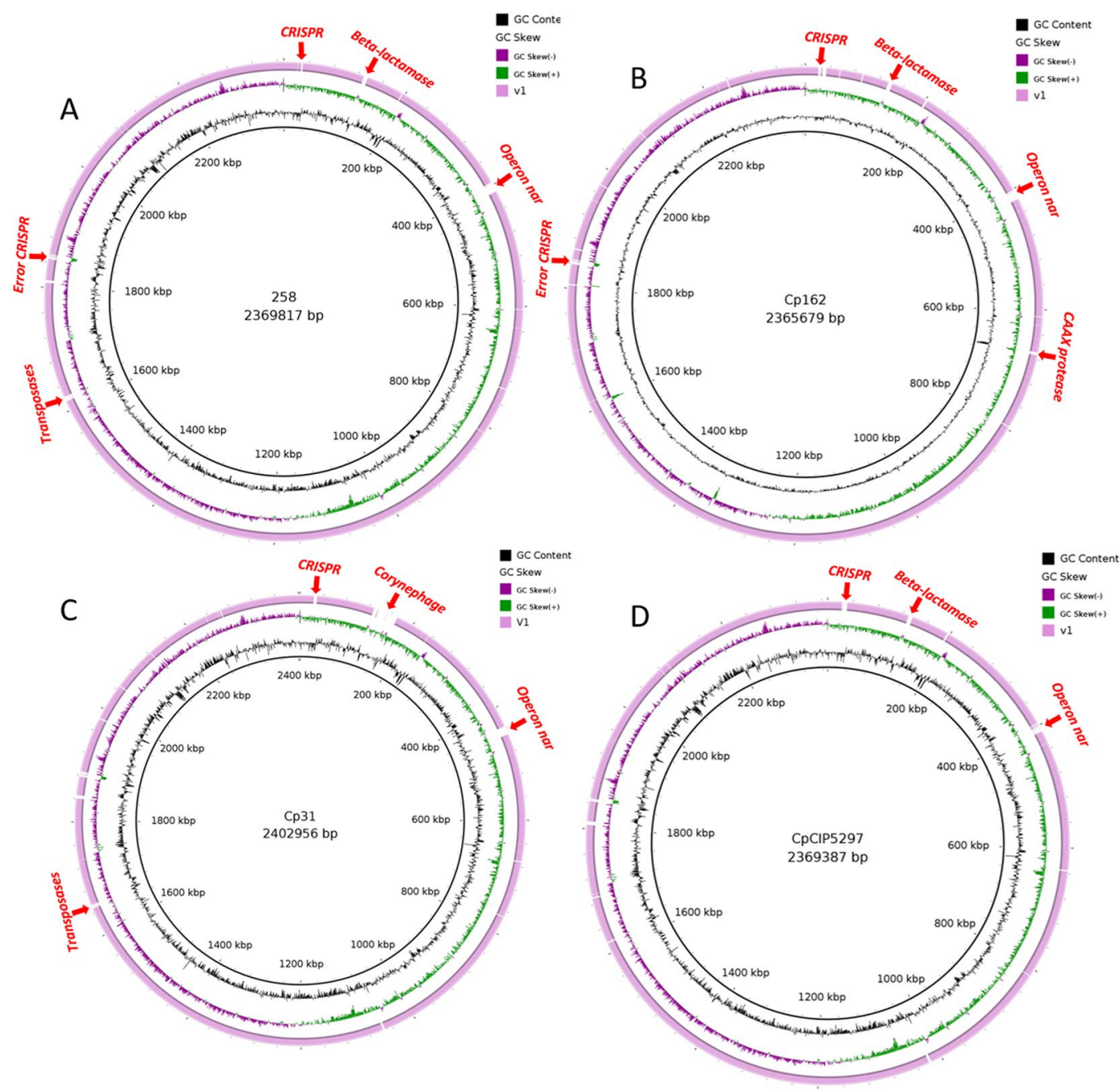

Figure 4. Comparative BRIG analysis of equi biovar strains. Comparative genomic maps of the older versions (outermost circles in purple) and their respective versions with optical map (inner black circles). (A) C. pseudotuberculosis 258. (B) C. pseudotuberculosis Cp162. (C) C. pseudotuberculosis 31. (D) C. pseudotuberculosis CIP52.97.

The CpCIP52.97 strain had an increase of $\sim 49 \mathrm{~kb}$, which represented a gain of 127 CDSs and the reduction of 13 pseudogenes (Table 2). In Fig. 4D, we can highlight the absence of essential genes, such as genes associated with CRISP (cas2, cas 1, cas3, cas4, cas5, cas6, cas7). Once more, the operon nar was absent, as well as genes coding for Beta-lactamase, Phytoene dehydrogenase, integrins, transposases, and several hypothetical proteins.

With the complete and finalized genomes, a multiple alignment analysis of the genome was done using Mauve. Figure 5 shows the genomes of 5 ovis strains (i.e., Cp1002B, Cp29156, CpFRC41, CpI19, and CpT1). Blocks with the same color represent conserved regions, in which they share a high similarity. Inversions and rearrangement events were established as changes in the $\mathrm{Cp} 1002 \mathrm{~B}$ reference synteny (first genome of the figure). By analyzing the ends of the inverted blocks, the inversions are flanked by clusters of rRNAs. Only the CpFRC41 and CpT1 strains showed the same gene order (synteny) in their genome. Using the same strategy to compare the equi strains (e.g., Cp31, Cp258, Cp162, CpCIP52.97, CpMB302, and MB11), the strain Cp162 was the only one that showed an inversion and rearrangements (Fig. 6). However, these blocks are not flanked by the rRNA clusters.

\section{Discussion}

Although the optical map initially focused on typing and identification of strains without the need of sequencing, it is an efficient approach to order contigs generated by de novo assembly, allowing error detection and an accurate contig ordering in assemblies ${ }^{30}$. By definition, the optical mapping technique is a single molecule barcode using restriction enzymes, and the distances among these restriction sites, which are the basis for the alignments between optical maps and the in-silico maps (by contigs or genomes). These features are excellent for the scaffolding process with de novo assembled contigs ${ }^{31}$. Onmus-Leone and collaborators in 2013 applied this technique to successfully order and correct contigs generated in the de novo assembly step using pyrosequencing data of the bacteria Providencia stuartii. The mentioned authors established the strategy of using the optical map to order and correct contigs generated from short reads ${ }^{32}$. 


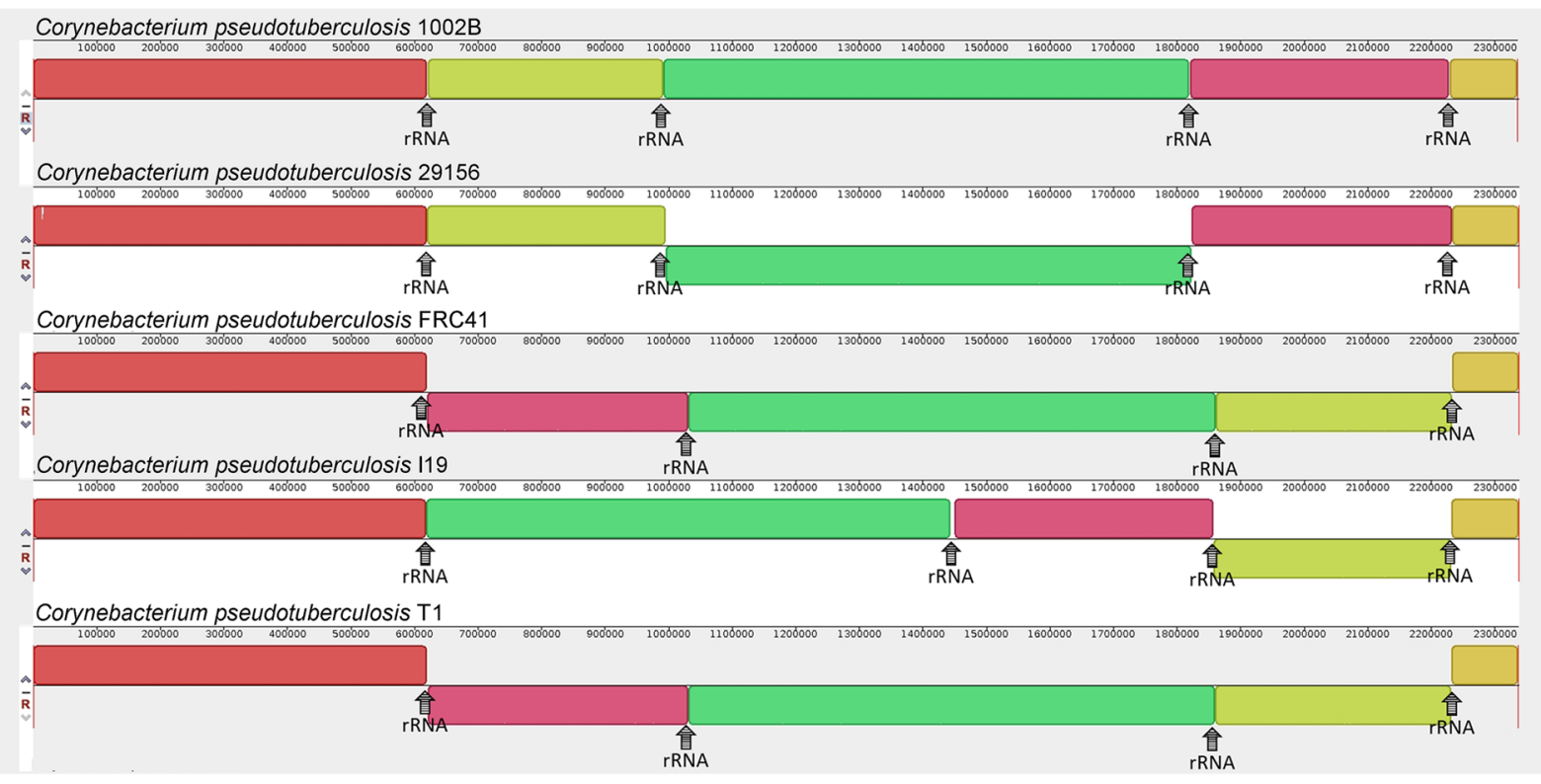

Figure 5. Comparative MAUVE analysis of ovis biovar strains. Comparison of Genome alignment of $C$. pseudotuberculosis 1002B, C. pseudotuberculosis 29156, C. pseudotuberculosis FRC41, C. pseudotuberculosis I19 and C. pseudotuberculosis T1 strains.

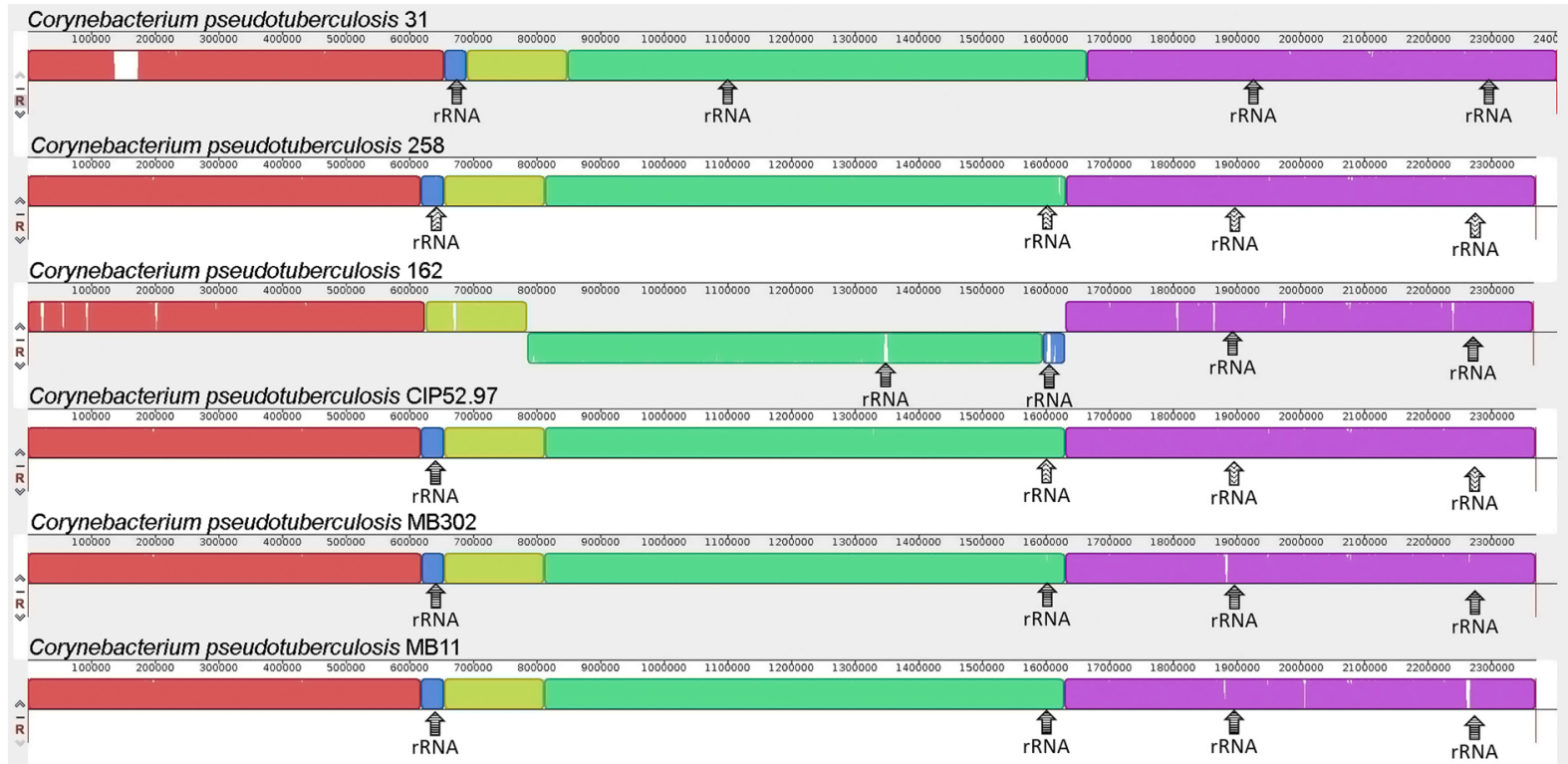

Figure 6. Comparative MAUVE analysis of equi biovar strains. Comparison of Genome alignment of $C$. pseudotuberculosis 31, C. pseudotuberculosis 258, C. pseudotuberculosis Cp162, C. pseudotuberculosis CIP52.97, C. pseudotuberculosis MB302 and C. pseudotuberculosis MB11.

Latreille and collaborators in 2007 highlighted the efficiency of the optical map suggesting it as a routine procedure in the assembly finishing process using de novo assembly ${ }^{33}$. According to these authors, it is possible to detect errors in the order and construction of the contigs by using this technique even when the organism presents several repeated regions. In the mentioned work, it was possible to finish the assembly by using optical map data even when cosmid libraries and overlapping restriction maps of BACs ${ }^{33}$ have already been applied without success. Analyzing these results, it was possible to conclude that the optical maps are an excellent option for bacterial assembly finishing because the restriction sites cover these repetitive regions in most cases.

Repetitive regions are considered a major difficulty for de novo assembly algorithms, mostly in transposons and ribosomal RNA cluster regions ${ }^{33}$. The strain Cp1002 was sequenced by using 454 Genome Sequencer FLX (Roche), Sanger e PacBio technologies, but the error of inversion in rRNA clusters continued. Only when 
sequencing with Ion Torrent $\mathrm{PGM}^{\mathrm{TM}}$ and ordering the contigs by using optical mapping data were done, the genome was correctly finished. The strain CpI19 was, at first, sequenced by using SOLiD v2 technology with $25 \mathrm{bp}$ mate-paired reads and a coverage depth of 321 -fold. The mate-paired technology may have contributed to the correct construction of the contigs, but due to its small size of reads sequenced, the inversion of the ribosomal RNA cluster regions occurred.

Trost and collaborators sequenced CpFRC41 strain in $2010^{34}$ by using 454 Genome Sequencer FLX (Roche) sequencer; it was the first C. pseudotuberculosis strain deposited in GenBank by NCBI in 2010. The assembly was performed using Corynebacterium diphtheriae NCTC 13129 (BX248353.1) as the reference genome, and the gaps were closed by using Polymerase Chain Reaction (PCR) and the software $\mathrm{r}^{\mathrm{cat}}{ }^{35}$. Gap filling by using PCR probably contributed to no inversions found in the final genome (Fig. 1D). Schröder and collaborators in 2011 successfully used this approach in Corynebacterium variabile DSM $44702^{36}$.

In CpMB11 strain (Fig. 1E) a standardization problem regarding the region of the $5^{\prime}$ end of the $d n a A$ gene was found. Several works have shown that the most common origin of replication in bacteria is oriC and the first gene is the chromosomal replication initiator protein DnaA $(d n a A)^{37}$. Thus, it is used as a standard pattern to linearize bacterial genomes.

Cp31 have been sequenced using several platforms: Solid v2 (CP003421.1), Ion Torrent PGM ${ }^{\mathrm{TM}}$ (CP003421.3) and PacBio. This fact confers more reliability to the assembly of this strain, which leads us to consider it as the reference strain in equi biovar. In its last version, published by Viana and collaborators in 2017, it was assembled using optical mapping technology. The strains Cp258, Cp162, Cp31 e CpCIP52.97 were initially sequenced in the SOLiD platform; these strains also belong to equi biovar and were characterized by using biochemical tests. Those were re-sequenced using Ion Torrent $\mathrm{PGM}^{\mathrm{TM}}$ platform and novel genomic regions were added to the genome sequence. Essential genes in regions, such as nar cluster and clusters associated with CRISPR proteins, which are only present in equi biovar, were added. The missing regions may be caused by an error propagation due to reference contig ordering, because of the first equi genome available, the $\mathrm{Cp} 258^{38}$ strain, was assembled using an ovis biovar strain as the reference, which does not completely present the referred clusters. The same issue happened to $\mathrm{Cp} 31^{39}$ isolate.

Similarly, the $\mathrm{Cp} 162^{40}$ strain presented the same issue because its contigs have been ordered according to $C$. pseudotuberculosis 316 (Cp316) (CP003077.1) ${ }^{41}$. Cp316 strain belongs to equi biovar and does not present nar operon in its former genomic sequence; it was assembled using the ovis strain CpFRC41 as a reference genome for contigs ordering. Presumably, the same problem happened in CpCIP52.97 $7^{42}$, but the genome used as a reference in the assembly process is not described in the article.

Using a complete genome deposited in public databases as a reference to assemble novel genomes is a risky strategy because even if it generates complete genomes more efficiently, it may disseminate assembly errors from one strain to other ${ }^{8}$. In this article, the optical map is used to validate contigs, and it is shown that extension and gap filling using read mapping or de novo assembly may generate assembly errors. We highlighted this technique because it does not present sequencing bias. Assembly statistics such as N50, coverage and depth coverage may generate false positive answers. Another strategy suggested by Lehri and collaborators is to use non-paired reads together with paired or long reads. A genomic region presenting assembly errors caused by insertions, deletions, inversions or rearrangements may hide significant biological variations or produce false interpretations, mostly in genomic analysis ${ }^{43}$. Even before the NGS platforms boomed, Schmutz and collaborators (2004) were concerned about the quality of the human genome ${ }^{43}$, mostly because of the possible assembly errors.

The inversions caused by RNA ribosomal clusters in ovis biovar strains may occur due to the high similarity of these clusters. This kind of inversion has already been shown in literature in bacteria as Salmonella paratyphi A using restriction enzymes and pulsed-field electrophoresis gel (PFGE ${ }^{44}$. The inversions among strains of the same species may be comprehended as homologous recombination events ${ }^{45}$. It can be highlighted that these inversions do not occur in strains belonging to equi biovar, except for Cp162. The inferred data concerning the genomic order of C. pseudotuberculosis strains were only achieved because optical mapping technology provides an accurate in vitro evidence.

\section{Methods}

Strain and DNA isolation and Genome sequencing. The methodology described below was applied to the novel sequencings of the strains C. pseudotuberculosis 1002(1002B), C. pseudotuberculosis 29156, C. pseudotuberculosis I19, C. pseudotuberculosis Cp162, C. pseudotuberculosis 258, C. pseudotuberculosis 31, C. pseudotuberculosis MB302, C. pseudotuberculosis MB11, C. pseudotuberculosis T1 and C. pseudotuberculosis CIP5297. The strains were cultivated in solid media with $1.5 \%$ of bacteriological agar. Subsequently, an isolated colony was used to grow in liquid media with brain-heart-infusion media (BHI-Hi Media Laboratories Pvt. Ltd, India) supplemented with $0.5 \%$ of Tween 80 , at $37^{\circ} \mathrm{C}$ for 20 hours under rotation. Genomic DNA was extracted following the protocol of Pacheco in $2006^{46}$. After the extraction step, the libraries were constructed with IonXpress ${ }^{\mathrm{TM}}$ Plus DNA Fragment Library Preparation Kit. The DNA samples were fragmented using Ion Shear ${ }^{\mathrm{TM}}$ Plus for five minutes at $37^{\circ}$. Then, adaptors from Ion Xpress ${ }^{\mathrm{TM}}$ Barcode Adapters kit were ligated for library quantification. Subsequently, the fragments were amplified using Ion PGM $^{\mathrm{TM}} 200 \mathrm{bp}$ or $400 \mathrm{bp}$ kits. These reactions were transferred to the semiconductor chip (ION $318^{\mathrm{TM}}$ Chip v2), and it was put into Ion PGM ${ }^{\mathrm{TM}}$. During all the steps described above, all the manufacturer's instructions were strictly followed. No novel sequencing was performed for C. pseudotuberculosis FRC41.

Genome assembly and annotation. The analysis of the reads quality was performed by using FastQC software (http://www.bioinformatics.babraham.ac.uk/projects/fastqc). No trimming was performed on reads with Phred score above 20, which were the majority. For contigs construction, we applied the de novo assembly strategy (no reference used) by using Mira v. 3.9.18 ${ }^{46}$, MIRA 4.0.2 ${ }^{46}$, Spades v. 3.6.0 ${ }^{47}$ e Newbler v. $2.9^{48}$ (Table 2) 


\begin{tabular}{|l|l|l|l|l|l|l|l|}
\hline Strains & Enzyme & Length (bp) & $\begin{array}{l}\text { Number of } \\
\text { fragments }\end{array}$ & $\begin{array}{l}\text { Average } \\
\text { fragment } \\
\text { size (bp) }\end{array}$ & $\begin{array}{l}\text { Maximum } \\
\text { fragment } \\
\text { size (bp) }\end{array}$ & $\begin{array}{l}\text { Minimum } \\
\text { fragment } \\
\text { size (bp) }\end{array}$ & $\begin{array}{l}\text { Whole } \\
\text { genome } \\
\text { coverage }\end{array}$ \\
\hline 1002B & Kpn1 & $2,335,144$ & 353 & $6,615.139$ & 38,715 & 903 & $99.998 \%$ \\
\hline 29156 & Kpn1 & $2,351,288$ & 368 & $6,389.37$ & 38,839 & 1,275 & $99.462 \%$ \\
\hline I19 & Kpn1 & $2,326,586$ & 333 & $6,986.745$ & 38,215 & 1,460 & $100.473 \%$ \\
\hline FRC41 & Kpn1 & $2,341,893$ & 369 & $6,346.593$ & 38,942 & 1,394 & $99.830 \%$ \\
\hline 31 & Kpn1 & $2,372,071$ & 346 & $6,855.697$ & 35,806 & 1,544 & $101.302 \%$ \\
\hline 162 & Kpn1 & $2,345,656$ & 362 & $6,479.713$ & 28,013 & 1,509 & $100.861 \%$ \\
\hline 258 & Kpn1 & $2,366,195$ & 346 & $6,838.714$ & 36,249 & 1,667 & $100.153 \%$ \\
\hline CIP52.97 & Kpn1 & $2,352,141$ & 347 & $6,778.504$ & 36,145 & 1,567 & $100.733 \%$ \\
\hline MB302 & Kpn1 & $2,363,709$ & 362 & $6,529.583$ & 36,517 & 1,471 & $100.215 \%$ \\
\hline T1 & Spe1 & $2,350,532$ & 193 & $12,178.922$ & 54,138 & 1,787 & $99.432 \%$ \\
\hline MB11 & Kpn1 & $2,347,572$ & 366 & $6,414.131$ & 36,155 & 1,333 & $100.679 \%$ \\
\hline
\end{tabular}

Table 3. Information about the quality metrics of the optical maps used.

software. Scaffolds construction was manually performed in CLC Genomics Workbench (CLC-gw) version 7.0 (Qiagen) software using the visualization of the contigs mapped and ordered according to the restriction sites of the strains in MapSolver ${ }^{\mathrm{TM}}$ (OpGen). Then, dnaA gene was fixed in the probable oriC position in the chromosome by using an in-house python script. In order to fill the gaps and finish the assembly, GapBlaster ${ }^{49}$ and FGAP ${ }^{50}$ software was used and subsequently, the contigs were mapped to the scaffold or a reference genome by using CLC Genomics (Qiagen). The annotation was performed by using in-house scripts for annotation transference from C. pseudotuberculosis strains, which were manually curated in the UniProt database (http://uniprot.org). Finally, pseudogenes were manually curated by using Artemis software ${ }^{51}$ and CLC Genomics (Qiagen).

Optical mapping. The optical maps were acquired from Opgen, Inc. The MapSolver ${ }^{\mathrm{TM}}$ (OpGen Inc.) software was used for the comparison of the physical restriction map and the restriction sites present in the assembled genome. Several pieces of information about metrics for the quality of each optical map are available in Table 3.

Genome plasticity and genetics content. This analysis was performed using genomic sequences before and after assembly assisted by optical mapping data. The maps comparing different versions of the studied strains were generated by using Blast Ring Image Generator (BRIG) v0.95 $5^{52}$. For inversion, deletion and rearrangement analysis, the Mauve v. 2.3.1 ${ }^{53}$ software was used with progressiveMauve ${ }^{53}$ option set.

\section{Conclusion}

The results obtained from optical mapping data analysis pointed errors in the assemblies of C. pseudotuberculosis genomes deposited in Genbank. Thus, the optical map was efficient in the assembly error detection of the strains Cp1002, CpI19, Cp31, Cp162, CpMB11, Cp258, and CpCIP52.97. Regarding the novel genomes, such as Cp29156, CpT1, and MB302, the optical map data contributed in the contigs ordering step, which contributed to a more efficient assembly finishing considering there are no assembly errors in the final version of the genomes. Furthermore, the update of the genomic sequences done by re-sequencing the genomes with Ion Torrent PGM ${ }^{\mathrm{TM}}$ platform was essential to the relevant genomic content increase, which happened in the strains previously sequenced using the SOLiD platform. We also pointed out several inversions caused by ribosomal RNA gene clusters in strains of the ovis biovar. Thus, we can suggest that the genomes deposited after applying this strategy made these strains more reliable for novel studies.

Received: 4 January 2019; Accepted: 18 October 2019;

Published online: 08 November 2019

\section{References}

1. Margulies, M. et al. Genome sequencing in microfabricated high-density picolitre reactors. Nature 437, 376-80 (2005).

2. Bentley, D. R. et al. Accurate whole human genome sequencing using reversible terminator chemistry. Nature 456, 53-59 (2008).

3. Rothberg, J. M. et al. An integrated semiconductor device enabling non-optical genome sequencing. Nature 475, 348-352 (2011).

4. Jain, M., Olsen, H. E., Paten, B. \& Akeson, M. The Oxford Nanopore MinION: delivery of nanopore sequencing to the genomics community. Genome Biol. 17, 256 (2016).

5. Bertsch, J. et al. GOLD:Genomes Online Database. GOLD Statistics (2018). Available at, https://gold.jgi.doe.gov/statistics. (Accessed: 8th August 2018).

6. Loman, N. J. et al. High-throughput bacterial genome sequencing: an embarrassment of choice, a world of opportunity. Nat. Rev. Microbiol. 10, 599-606 (2012).

7. Lehri, B., Seddon, A. M. \& Karlyshev, A. V. The hidden perils of read mapping as a quality assessment tool in genome sequencing. Sci. Rep. 7, 43149 (2017).

8. Narzisi, G. \& Mishra, B. Comparing De Novo genome assembly: The long and short of it. PLoS One 6 (2011).

9. Boetzer, M., Henkel, C. V., Jansen, H. J., Butler, D. \& Pirovano, W. Scaffolding pre-assembled contigs using SSPACE. Bioinformatics 27, 578-9 (2011).

10. Mariano, D. C. et al. MapRepeat: an approach for effective assembly of repetitive regions in prokaryotic genomes. Bioinformation 11(6), 276-279 (2015).

11. Waters, N. R., Abram, F., Brennan, F., Holmes, A. \& Pritchard, L. riboSeed: leveraging prokaryotic genomic architecture to assemble across ribosomal regions. Nucleic Acids Res. 46, e68-e68 (2018). 
12. Wu, C., Schramm, T. M., Zhou, S., Schwartz, D. C. \& Talaat, A. M. Optical mapping of the Mycobacterium avium subspecies paratuberculosis genome. BMC Genomics 10, 25 (2009).

13. Schwartz, D. C. et al. Ordered restriction maps of Saccharomyces cerevisiae chromosomes constructed by optical mapping. Science 262, 110-4 (1993).

14. Samad, A., Huff, E. F., Cai, W. \& Schwartz, D. C. Optical mapping: a novel, single-molecule approach to genomic analysis. Genome Res. 5, 1-4 (2007).

15. Reslewic, S. et al. Whole-Genome Shotgun Optical Mapping of Rhodospirillum rubrum Whole-Genome Shotgun Optical Mapping of Rhodospirillum rubrum. Appl. Environ. Microbiol. 2005 71, 5511 (2005).

16. Kotewicz, M. L., Mammel, M. K., LeClerc, J. E. \& Cebula, T. A. Optical mapping and 454 sequencing of Escherichia coli O157: H7 isolates linked to the US 2006 spinach-associated outbreak. Microbiology 154, 3518-3528 (2008).

17. Petersen, R. F. et al. Molecular Characterization of Salmonella Typhimurium Highly Successful Outbreak Strains. Foodborne Pathog. Dis. 8, 655-661 (2011).

18. Sabirova, J. S., Xavier, B. B., Ieven, M., Goossens, H. \& Malhotra-Kumar, S. Whole genome mapping as a fast-track tool to assess genomic stability of sequenced Staphylococcus aureus strains. BMC Res. Notes 7, 1-6 (2014).

19. Shukla, S. K. et al. Comparative whole-genome mapping to determine Staphylococcus aureus genome size, virulence motifs, and clonality. J. Clin. Microbiol. 50, 3526-3533 (2012).

20. Zhou, S. et al. A whole-genome shotgun optical map of Yersinia pestis strain KIM. Appl. Environ. Microbiol. 68, 6321-6331 (2002).

21. Zhou, S. Whole-Genome Shotgun Optical Mapping of Rhodobacter sphaeroides strain 2.4.1 and Its Use for Whole-Genome Shotgun Sequence Assembly. Genome Res. 13, 2142-2151 (2003).

22. Lin, J. Whole-Genome Shotgun Optical Mapping of Deinococcus radiodurans. Science. 285, 1558-1562 (1999).

23. Olsen, R. A. et al. De novo assembly of Dekkera bruxellensis: a multi technology approach using short and long-read sequencing and optical mapping. Gigascience 4, 56 (2015).

24. Chamala, S. et al. Assembly and validation of the genome of the nonmodel basal angiosperm Amborella. Science. 342, 1516-1517 (2013).

25. Zhou, S. et al. Validation of rice genome sequence by optical mapping. BMC Genomics 8, 278 (2007).

26. Tang, H., Lyons, E. \& Town, C. D. Optical mapping in plant comparative genomics. Gigascience 4, 1-6 (2015).

27. Dong, Y. et al. Sequencing and automated whole-genome optical mapping of the genome of a domestic goat (Capra hircus). Nat. Biotechnol. 31, 135-41 (2013).

28. Mariano, D. C. B. et al. Whole-genome optical mapping reveals a mis-assembly between two rRNA operons of Corynebacterium pseudotuberculosis strain 1002. BMC Genomics 17, 1-7 (2016).

29. Oliveira, A. et al. Insight of Genus Corynebacterium: Ascertaining the Role of Pathogenic and Non-pathogenic Species. Front. Microbiol. 8, 1937 (2017).

30. Bogas, D. et al. Applications of optical DNA mapping in microbiology. Biotechniques 62, 255-267 (2017).

31. Mendelowitz, L. \& Pop, M. Computational methods for optical mapping. Gigascience 3, 1-7 (2014).

32. Onmus-Leone, F. et al. Enhanced De Novo Assembly of High Throughput Pyrosequencing Data Using Whole Genome Mapping. PLoS One 8, 2-10 (2013).

33. Latreille, P. et al. Optical mapping as a routine tool for bacterial genome sequence finishing. BMC Genomics 8, 321 (2007).

34. Trost, E. et al. The complete genome sequence of Corynebacterium pseudotuberculosis FRC41 isolated from a 12-year-old girl with necrotizing lymphadenitis reveals insights into gene-regulatory networks contributing to virulence. BMC Genomics 11, 728 (2010).

35. Husemann, P. \& Stoye, J. r2cat: Synteny plots and comparative assembly. Bioinformatics 26, 570-571 (2009).

36. Schröder, J., Maus, I., Trost, E. \& Tauch, A. Complete genome sequence of Corynebacterium variabile DSM 44702 isolated from the surface of smear-ripened cheeses and insights into cheese ripening and flavor generation. BMC Genomics 12, 545 (2011).

37. Eisen, J. A., Heidelberg, J. F., White, O. \& Salzberg, S. L. Evidence for symmetric chromosomal inversions around the replication origin in bacteria. Genome Biol. 1, RESEARCH0011 (2000).

38. Soares, S. C. et al. Genome sequence of Corynebacterium pseudotuberculosis biovar equi strain 258 and prediction of antigenic targets to improve biotechnological vaccine production. J. Biotechnol. 167, 135-41 (2013).

39. Silva, A. et al. Complete genome sequence of Corynebacterium pseudotuberculosis Cp31, isolated from an Egyptian buffalo. J. Bacteriol. 194, 6663-6664 (2012).

40. Hassan, S. S. et al. Whole-genome sequence of Corynebacterium pseudotuberculosis strain Cp162, isolated from camel. J. Bacteriol. 194, 5718-5719 (2012)

41. Ramos, R. T. J. et al. Genome Sequence of the Corynebacterium pseudotuberculosis Cp316 Strain, Isolated from the Abscess of a Californian Horse. J. Bacteriol. 194, 6620-6621 (2012).

42. Cerdeira, L. T. et al. Complete genome sequence of Corynebacterium pseudotuberculosis strain CIP 52.97, isolated from a horse in Kenya. J. Bacteriol. 193, 7025-7026 (2011).

43. Salzberg, S. L. \& Yorke, J. A. Beware of mis-assembled genomes. Bioinformatics 21, 4320-4321 (2005).

44. Liu, S. L. \& Sanderson, K. E. The chromosome of Salmonella paratyphi A is inverted by recombination between $\mathrm{rrnH}$ and $\operatorname{rrnG}$. J. Bacteriol. 177, 6585-6592 (1995).

45. Raeside, C. et al. Large Chromosomal Rearrangements during a Long-Term Evolution Experiment with Escherichia coli. MBio 5, e01377-14 (2014)

46. Chevreux, B., Wetter, T. \& Suhai, S. Genome Sequence Assembly Using Trace Signals and Additional Sequence Information. Comput. Sci. Biol. Proc. Ger. Conf. Bioinforma, 45-56, 10.1.1.23/7465 (1999).

47. Bankevich, A. et al. SPAdes: A New Genome Assembly Algorithm and Its Applications to Single-Cell Sequencing. J. Comput. Biol. 19, 455-477 (2012)

48. 454 Life Sciences Corp. Local Newbler 2.9 documentation, https://hpc.wm.edu/software/docs/newbler/index.html (2013).

49. de Sá, P. H. C. G. et al. GapBlaster-A Graphical Gap Filler for Prokaryote Genomes. PLoS One 11, e0155327 (2016).

50. Piro, V. C. et al. FGAP: an automated gap closing tool. BMC Res. Notes 7, 371 (2014).

51. Carver, T., Harris, S. R., Berriman, M., Parkhill, J. \& McQuillan, J. A. Artemis: An integrated platform for visualization and analysis of high-throughput sequence-based experimental data. Bioinformatics 28, 464-469 (2012)

52. Alikhan, N. F., Petty, N. K., Ben Zakour, N. L. \& Beatson, S. A. BLAST Ring Image Generator (BRIG): Simple prokaryote genome comparisons. BMC Genomics 12, 402 (2011)

53. Darling, A. E., Mau, B. \& Perna, N. T. progressiveMauve: Multiple Genome Alignment with Gene Gain, Loss and Rearrangement. PLoS One 5, el1147 (2010).

54. Mariano, D. C. B., Ramos, R. T. J. \& Azevedo, V. A. D. C. Montagem e finalização de genomas procariotos com mapeamento óptico. Novass $\mathbf{7 6}$ (2016).

55. Viana, M. V. C. et al. Comparative genomic analysis between Corynebacterium pseudotuberculosis strains isolated from buffalo. PLoS One 12, e0176347 (2017).

56. Mariano, D. C. B. et al. SIMBA: A web tool for managing bacterial genome assembly generated by Ion PGM sequencing technology. BMC Bioinformatics 17, 456 (2016).

57. Baraúna, R. A. et al. Assessing the Genotypic Differences between Strains of Corynebacterium pseudotuberculosis biovar equi through Comparative Genomics. PLoS One 12, e0170676 (2017). 
58. Almeida, S. et al. Complete Genome Sequence of the Attenuated Corynebacterium pseudotuberculosis Strain T1. Genome Announc. 4, e00947-16 (2016).

59. Barauna, R. A. et al. Genomic analysis of four strains of Corynebacterium pseudotuberculosis bv. equi isolated from horses showing distinct signs of infection. Stand. Genomic Sci. 12, 16 (2017).

\section{Acknowledgements}

The author's thanks CNPq, FAPEMIG, CAPES, and PRPq by the support. This work was supported by Conselho Nacional de Desenvolvimento Científico e Tecnológico (CNPq), Fundação de Amparo à Pesquisa do Estado de Minas Gerais (FAPEMIG), Coordenação de Aperfeiçoamento de Pessoal de Nível Superior (CAPES) and PróReitoria de Pesquisa da Universidade Federal de Minas Gerais (PRPq).

\section{Author contributions}

T.d.J.S. performed genome assemblies, optical mapping analysis, interpreted all data regarding and was a major contributor in writing the manuscript. D.P. and M.T.D.P. performed genome assemblies, optical mapping analysis, and were contributors in writing the manuscript. R.P. performed content and genomes plasticity analysis and was a contributor in writing the manuscript. F.L.P., H.C.P.F., and B.B. performed genome sequencing and were contributors in writing the manuscript. A.C.P.G., R.B.K., R.R., A.S., P.G., D.B., A.G.N., V.A. contributed to data interpretation and were major contributors in writing the manuscript.

\section{Competing interests}

The authors declare that the research was conducted in the absence of any commercial, financial or nonfinancial relationships that could be construed as a potential conflict of interest.

\section{Additional information}

Correspondence and requests for materials should be addressed to V.A.

Reprints and permissions information is available at www.nature.com/reprints.

Publisher's note Springer Nature remains neutral with regard to jurisdictional claims in published maps and institutional affiliations.

(c) Open Access This article is licensed under a Creative Commons Attribution 4.0 International License, which permits use, sharing, adaptation, distribution and reproduction in any medium or format, as long as you give appropriate credit to the original author(s) and the source, provide a link to the Creative Commons license, and indicate if changes were made. The images or other third party material in this article are included in the article's Creative Commons license, unless indicated otherwise in a credit line to the material. If material is not included in the article's Creative Commons license and your intended use is not permitted by statutory regulation or exceeds the permitted use, you will need to obtain permission directly from the copyright holder. To view a copy of this license, visit http://creativecommons.org/licenses/by/4.0/.

(C) The Author(s) 2019 